\title{
ARE DWARF SPHEROIDAL GALAXIES DARK MATTER DOMINATED OR REMNANTS OF DISRUPTED LARGER SATELLITE GALAXIES? - A POSSIBLE TEST.
}

\author{
RALF S. KLESSEN ${ }^{1,2}$ AND HONGSHENG ZHAO ${ }^{3}$ \\ Accepted for publication in ApJ Letters
}

\begin{abstract}
The failure of standard cosmolocical models in accounting for the statistics of dwarf satellites and the rotation curve of gas-rich dwarf galaxies in detail has led us to examine whether earlier non-equilibrium models of dwarf spheroidal satellites without any dark matter should be reconsidered in more detail. Such models can explain the high dispersion of the dwarf spheroids by the projection of disrupted tidal debris. We show in the case of Milky Way satellites, that these models predict a significant spread in the apparent magnitude of horizontal branch stars which is correlated with sky position and velocity. In particular, the models produce a strong correlation of radial velocity with the long axis of the dwarf. Current data do not set strong enough constraint on models, but we suggest that photometric and spectroscopic surveys of extra-tidal stars of nearby dwarf spheroids in the Milky Way and Andromeda can falsify these models without dark matter.
\end{abstract}

Subject headings: galaxies: formation — galaxies: kinematics and dynamics — galaxies: photometry — galaxies: structure - Local Group

\section{INTRODUCTION}

At least nine dwarf spheroidal galaxies (dSph's) are known to orbit the Milky Way at distances ranging from a few tens to a few hundred kpc. Their velocity dispersions and stellar masses are similar to those seen in globular clusters, however, they are about two orders of magnitude more extended (e.g. Irwin \& Hatzidimitriou 1995, Grebel 1997, Mateo 1998). Under the standard assumption of virial equilibrium the derived mass-to-light ratios are extremely high (up to $M / L \approx 100$ ) implying the presence of huge quantities of dark matter. However, the assumption of virial equilibrium may not be valid for satellite galaxies: they could be significantly perturbed by Galactic tides or even not be bound at all, but instead be the remnants of disrupted larger satellite galaxies. Indeed, at least one dSph (Sagittarius, see Ibata, Gilmore, \& Irwin 1994) is known to be torn apart by tidal interaction with the Milky Way and others appear heavily distorted (e.g. Ursa Minor, Martínez-Delgado et al. 2001a).

The non-equilibrium models have received little attention partly because there is plenty of evidence for dark matter in halos of high-brightness galaxies from the study of, e.g., gravitational lensing. It appears unnecessary to explain the missing dynamical matter in dwarfs with a different theory. However, recent studies show that it is problematic to introduce dark matter in dwarfs (e.g. van den Bosch et al. 2000, McGaugh \& de Blok 2001), and in fact many have tried to remove the undesirable cusp in cold dark matter models by including feedback from baryonic material and from massive black holes or introducing modifications to the gravitational force law (Mateo 1998). It is thus interesting to reexamine options without dark matter to start with in dwarf galaxies.

The 'tidal scenario' has been studied by a variety of authors (e.g. Kuhn 1993, Oh, Lin \& Aarseth 1995, Piatek \& Pryor 1995, Helmi \& White 1999, Johnston, Sigursson, \& Hernquist 1999, Bekki, Cough, \& Drinkwater 2001). In particular Kroupa (1997, hereafter K97) and Klessen \& Kroupa (1998, hereafter KK98) have followed the long-term evolution of low- mass satellite galaxies in the Galactic tidal field in a series of high-resolution numerical simulations. A remnant containing a few percent of the initial mass prevails as a long-lived and distinguishable entity for a period of several billion years after the initial satellite dissolves. They proposed that what appears to be a bound dSph galaxy to an terrestrial observer may in fact be this elongated tidal remnant. This model successfully demonstrates that high velocity dispersions in dSphs may be obtained without any internal dark matter. And it accounts for the distorted morphology of e.g., the double-peaked Ursa Minor, as well (Kleyna et al. 1998). It is surprising such an extreme model passes the known observational test so well. It is the aim of this paper to give a first discussion of additional tests to falsify/confirm such models.

\section{NUMERICAL MODEL}

We follow the evolution and disintegration of a satellite dwarf galaxy in the tidal field of our Galaxy. The satellite has initially a mass of $10^{7} \mathrm{M}_{\odot}$, and a Plummer law density profile with core radius $R_{\mathrm{c}}=0.3 \mathrm{kpc}$ and truncation radius $R_{\mathrm{t}}=1.5 \mathrm{kpc}$. The central velocity dispersion in equilibrium is $\sigma_{\mathrm{c}}=4.4 \mathrm{~km} \mathrm{~s}^{-1}$. The stars have a mass-to-light ratio $M / L=3$ and there is no dark matter component in the dSph. The Milky Way, however, does contain dark matter. It dominates the gravitational potential at large scales. We describe it as isothermal sphere with circular velocity of $220 \mathrm{~km} \mathrm{~s}^{-1}$, and adopt a core radius of $5 \mathrm{pc}$ to obtain a total halo mass of $2.85 \times 10^{12} \mathrm{M}_{\odot}$ within a distance of $250 \mathrm{kpc}$.

The satellite galaxy consists of 131072 particles. The equation of motion is solved by direct summation using the specialpurpose hardware device GRAPE-3 (Sugimoto et al. 1990, Ebisuzaki et al. 1993) under the influence of a external rigid Galactic potential. For further details see KK98.

Here we focus on two model galaxies. Sat-M1 is on a highly eccentric orbit with $\epsilon=0.71$ and Sat-M2 has a more circular trajectory with $\epsilon=0.46$ (Table 1 in KK98). We follow their evolution for many orbital periods well into the phase when

\footnotetext{
${ }^{1}$ UCO/Lick Observatory, University of California at Santa Cruz, CA 95064, U.S.A. (ralf@ucolick . org)

${ }^{2}$ Max-Planck-Institut für Astronomie, Königstuhl 17, 69117 Heidelberg, Germany

${ }^{3}$ Institute of Astronomy, Cambridge, CB3 0HA, UK (hs zeast. cam.ac.uk)
} 

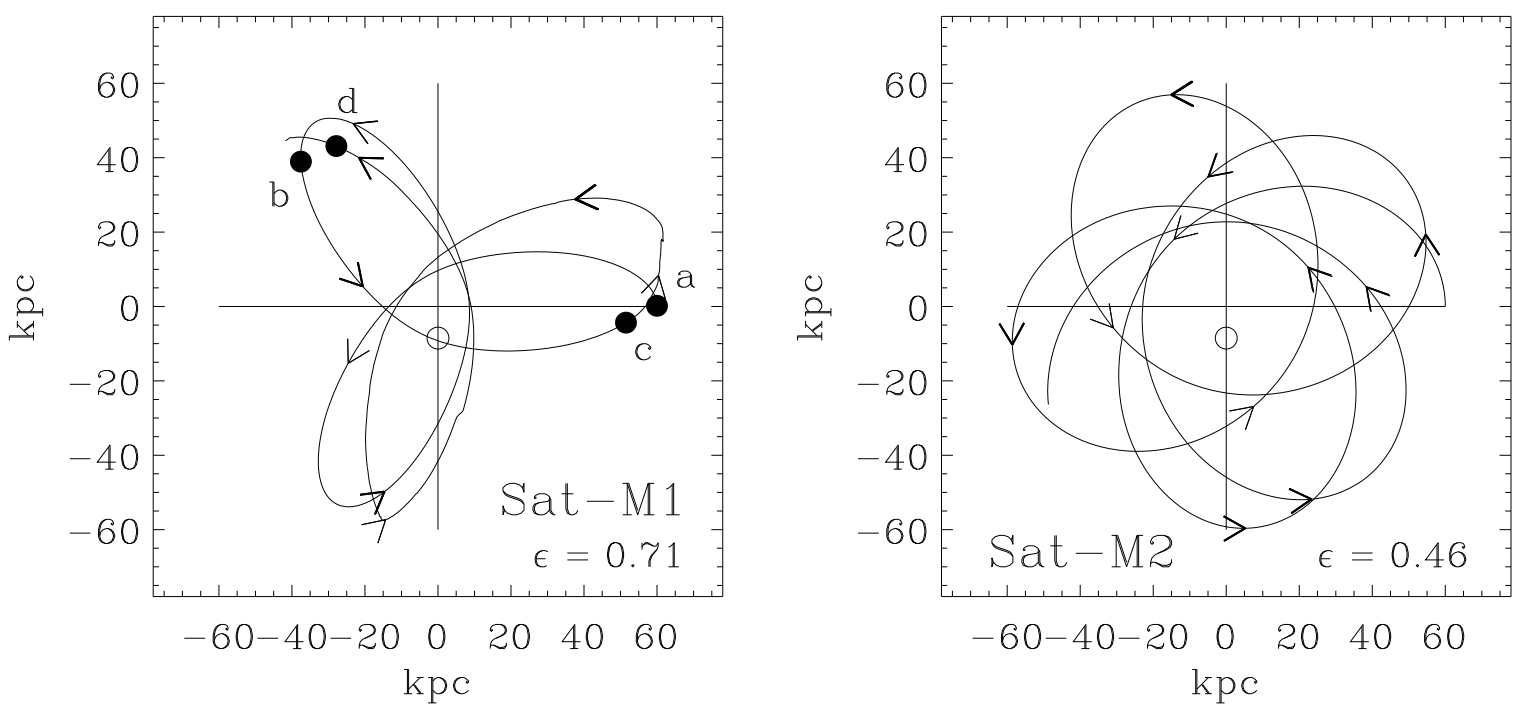

FIG. 1.- Center-of-density trajectorjes of the two dSph galaxies Sat-M1 and Sat-M2. The arrows indicate indicate time intervals of 500 Gyr. For Sat-M1, the four snapshots shown in Figures 3 , and 5 are indicated by filled dots. The position of the "observer on Earth" is indicate by a open circle.

the satellites are completely dissolved by tidal shocking. The dwarf galaxies are initially placed at a galactocentric distance of $60 \mathrm{kpc}$ with tangential velocities of $60 \mathrm{~km} \mathrm{~s}^{-1}$ and $100 \mathrm{~km} \mathrm{~s}^{-1}$, respectively (see Table 1 in KK98). The trajectories of the stellar density maximum are depicted in Figure 1. The position of a hypothetical observer on "Earth" is indicated by the open circle.

\section{SYNTHETIC CMD'S}

Studying the effect of distance variations on the distribution of stars in the color-magnitude diagram (CMD) requires a template CMD that is well defined and free of additional bias. Instead of constructing CMD's from population synthesis models, we found it more appropriate to use data of a well observed existing astrophysical object and chose the Galactic globular cluster M3 (i.e. NGC 5272). Globular clusters are massive but compact in size and their stars are more or less coeval. Hence, their CMD's are well populated and not influenced by variations in the stellar distance moduli or by a complicated star formation history. M3 is particularly well suited. It is a classical prototype of an old (Pop II) cluster. It is a well observed object with very accurate photometric data available. Bounanno et al. (1994) reanalyzed the Mt. Palomar and Mt. Wilson original plates (Sandage 1953) to obtain a sample of more than 10000 M3 stars with $V>21.5$. We use their data to construct our templated CMD.

M3 has a very thin and well defined $\mathrm{HB}$ with a large range in colors, $-0.4 \lesssim(B-V) \lesssim 0.6$ (see also Sandage \& Katem 1982). The faint end of the HB lies at $V \approx 17.5$ with photometric errors of typically $\lesssim 0.02 \mathrm{mag}$. The average "thickness" of the HB is $\Delta V \approx 0.08$, defined as the standard deviation from the mean value. The distance modulus of the cluster is $(m-M)_{0}=14.94$. Age and metallicity are estimated as $t=18.7 \pm 3.5 \mathrm{Gyr}$ and $\langle[\mathrm{Fe} / \mathrm{H}]\rangle=-1.66 \pm 0.10$. M3 falls therefore right into the range relevant for the Galactic dSph's.

To construct synthetic CMD's from the numerical models of $\mathrm{dSph}$ galaxies without dark matter, we use the CMD of M3 as starting point and select all stars with $V \leq 18$. Then we take the model galaxy, project it onto the sky of an hypothetical observer on "Earth". This procedure is introduced in K97 and KK98. The synthetic CMD of the dSph galaxy finally is obtained by convolving its stars with the template CMD using the appropriate distance moduli, i.e. for each star in the model galaxy we randomly select one star in the template CMD and modify its apparent luminosity according to its distance to the observer.

\section{HORIZONTAL BRANCH WIDTH}

Figure 2 illustrates what a terrestrial observer would "see" on the sky when observing Sat-MI at four different stages of its evolution. We depict the satellite when it is close to apogalacticon, as due to its eccentric orbit, this is where the satellite spends most of its time and is most likely to be observed. Each image shows a $10^{\circ} \times 10^{\circ}$ field on the sky centered on the stellar density maximum. After the second perigalactic passage in the tidal field of the Milky Way (i.e. after $t \gtrsim 1.5 \times 10^{9} \mathrm{yr}$ ) the satellite becomes completely unbound, but remains visible to the terrestrial observer as diffuse enhancement of stellar density for several billion years. This remnant looks remarkably similar to the dSph galaxies observed today. If virial equilibrium is assumed, the measured central LOS velocity dispersion $\sigma_{\mathrm{c}}$ implies $M / L$ ratios much larger than the true value $M /\left.L\right|_{\text {true }}=3$ (see Figure 6 in KB98).

The satellite remnant in Figure 2 is quite elongated and aligned with the orbital trajectory. As the satellite dissolves and populates extended tidal tails, the density maximum of the observed dSph traces the trajectory of the original satellite and fades in time as stars drift apart along the tidal streams. The elongated morphological appearence of the remnant for most of its orbit is the result of this tidal debris seen in projection. Besides the isopletic contours of the remnant from star counts, Figure 2 shows individual stars in the figure color-coded according to their distance moduli relative to the center. There are systematic distance variations along the semi-major axis, i.e. along the orbital path. The effect is clearly noticeable in the 

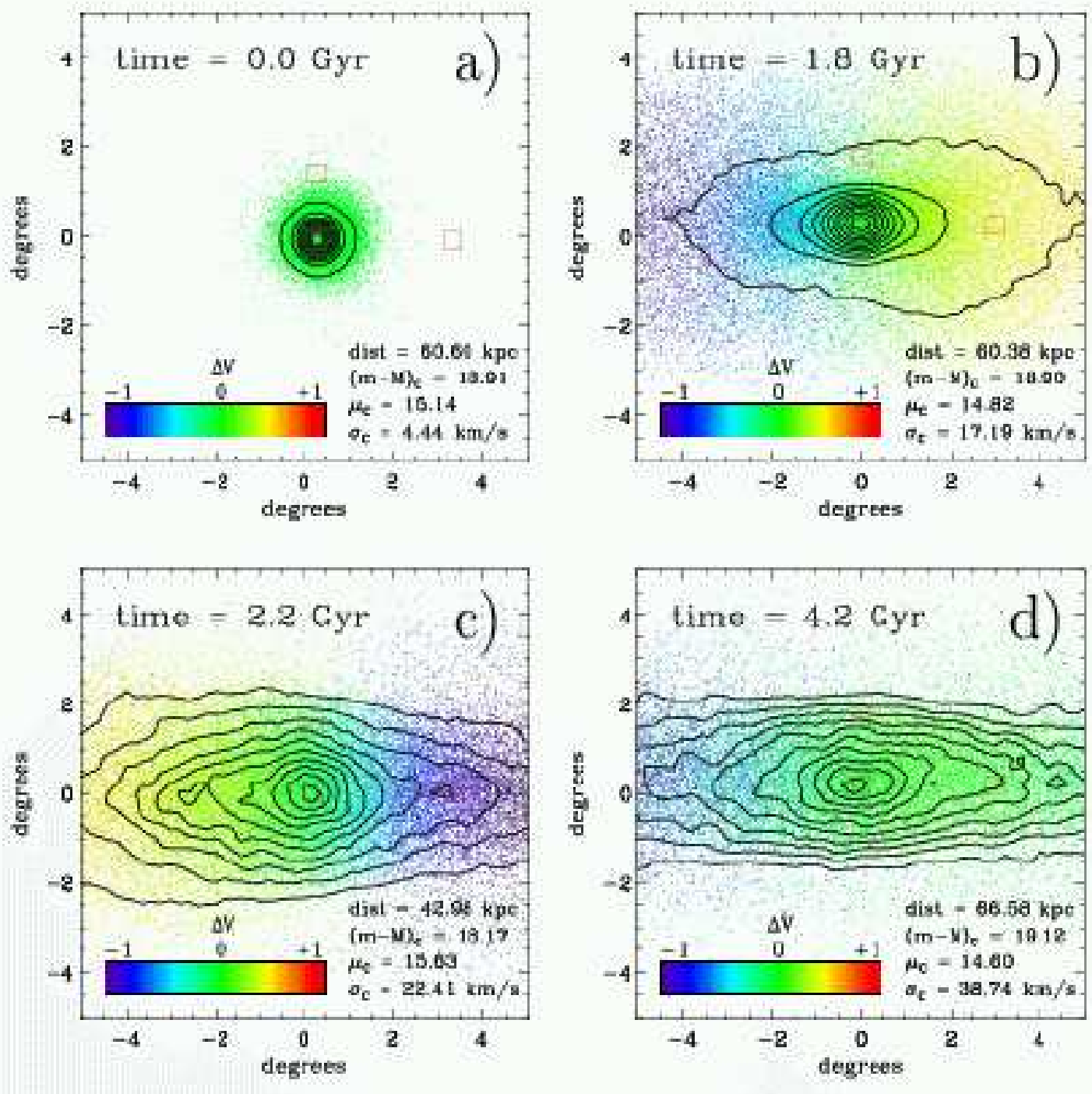

FIG. 2.- The model dwarf galaxy Sat-MI as an observer on Earth would see it on the sky at four different times of its evolution. The first image (a) shows the initial equilibrium Plummer sphere at a distance of $60.6 \mathrm{pc}$. In (b), the satellite has just gone through the second apogalacticon, and (c) it has passed through the third perigalacticon and approaches again an apogalacticon. Finally, in (d), the dSph galaxy is close to the fifth apogalacticon. The corresponding evolutionary time is indicated in the top part of each plot. The contour lines indicate the number density of stars, with a line spacing equal to $1 / 11$ of the measured dynamic range of the linear number density. Each star in the image is color-coded by the shift in $V$-magnitude due to distance modulus variations relative to the distance modulus $(m-M)_{\mathrm{c}}$ of the center-of-mass. Its distance and the corresponding value $(m-M)_{\mathrm{c}}$ are indicated in the lower part of the plot. Also the observed central surface brightness $\mu_{\mathrm{c}}$ and the central line-of-sight velocity dispersion $\sigma_{\mathrm{c}}$ is listed. Red boxes indicate the three sub-fields of size $0.5^{\circ} \times 0.5^{\circ}$ for which CMD's are obtained.

full $10^{\circ} \times 10^{\circ}$ field, i.e. when imaging the entire galaxy beyond several half light radii. The distance variations are difficult to detect in small fields. This demonstrates the necessity for wide field imaging surveys of the Milky Way satellite galaxies. To illustrate this, three sub-fields of $0.5^{\circ} \times 0.5^{\circ}$ are considered as well, one at the center of the dSph, one off by $3^{\circ}$ along the orbital path, and the other one off by $1.5^{\circ}$ perpendicular to that.

At this point, a note of caution is necessary. The two model galaxies discussed here resemble the observed Galactic dSph's in many aspects (see KK98), however, their surface density profiles are somehow shallower and more extended than typical observed ones. There are several reasons for this. (1) To directly build up on the previous investigation of KK98, we consider two models with apogalactic distances of only $60 \mathrm{kpc}$. The dwarf galaxies are relatively close to the hypothetical observer on Earth (who is at a galactic distance of $8.5 \mathrm{kpc}$ ) and their projections on his/her plane of the sky appear therefore relatively large. The Galactic dSph's all have larger distances and subsequently smaller apparent angular diameter. (2) Already in their initial configuration (see \$2 2 ), the two model galaxies are more extended than most observed dSph's. During tidal disruption they only spread out even further. (3) The considered satellite galaxies both have an initial mass of $10^{7} \mathrm{M}_{\odot}$. Given that the remnant only contains a few percent of the total mass, this starting value may be a bit small compared to most observed dSph's. (4) The orbital eccentricities of the model galaxies are only moderately high. However, the most compact morphology in projection is obtained in the tidal model for viewing angles close to the tangent to the orbit. As then the long axis of the tidal stream becomes normal to the plane of the sky, and stars 


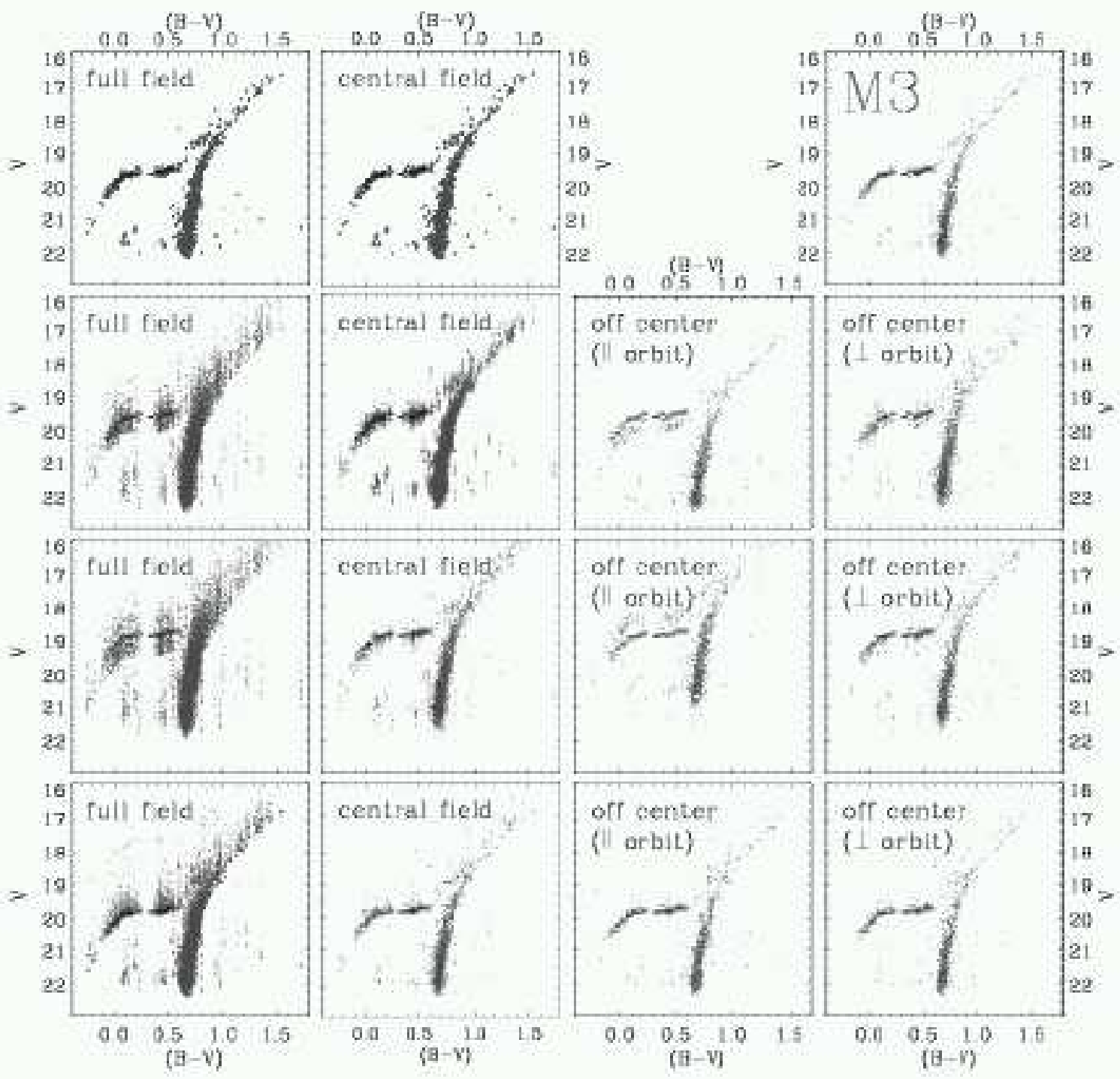

FIG. 3.- Synthetic CMD of the dwarf galaxy Sat-M1 as observed on "Earth" at four different stages of its dynamical evolution. The upper panel shows the initial configuration at $t=0$. The second row depicts the system immediately after the second apogalacticon, i.e. at $t=1.8 \mathrm{Gyr}$, third row indicates $t=2.3 \mathrm{Gyr}$ shortly before the third apogalacticon, and the bottom panel describes the dwarf galaxy at $t=4.0 \mathrm{Gyr}$ before its fifth apogalaction. The left column gives the CMD of the entire $5^{\circ} \times 5^{\circ}$ field shown in Figure 2 the second column focuses on a $0.5^{\circ} \times 0.5^{\circ}$ field in the center of the dSph galaxy, the third column gives the CMD from a $0.5^{\circ} \times 0.5^{\circ}$ field off-center by $3^{\circ}$ towards the West (along the orbital trajectory of the satellite galaxy), and the CMD's in the fourth column are obtained from a $0.5^{\circ} \times 0.5^{\circ}$ field shifted $1.5^{\circ}$ to the North (perpendicular to the orbital plane), as indicated by the red boxes in Figure 2 For comparison the CMD of the globular cluster M3 is plotted in the upper right corner of the figure (the off-center sub-fields at $t=0$ contain no stars). To guide your eye for estimating the effect of distance variations within the tidal galaxy, the HB position and width of M3 are indicated in the individual CMD's of the dSph (assuming the globular cluster would lie at a similar distance as the center of density of the dwarf).

basically "pile up" along the line of sight without strong excursions perpendicular to it. The object has maximal depth and minimal extent on the sky. Therefore, dwarf spheroidal galaxies that are very compact and exhibit a lack of significant numbers of extra-tidal stars (like Draco, see Odenkirchen et al. 2001, or Aparicio et al. 2001) must originate from initial satellites that move on highly eccentric orbits. This defines an additional test for the tidal models once reliable proper motions for the dSph's of the Milky Way become available. A study aimed at describing in detail individual Galactic satellites is beyond the scope of this paper. It requires an extensive (and expensive) investigation of large areas of the parameter space, and therefore remains as work for the future.

The resulting CMD's for the full model galaxy Sat-MI and for the three sub-fields are shown in Figure 3. To clearly detect any HB thickening in the CMD due to distance effects one needs to consider the wide-field image. Only immediately after the complete disruption of the satellite, i.e. when the stars have not had enough time to disperse significantly, is the stellar density still high enough to yield a statistically significant result in the central field. At later times and for the off-center sub-fields this is not the case, the HB does not appear significantly widened. It is, however, noticeable that the sub-fields which are displaced along the major axis (i.e. along the orbital trajectory) exhibit HB's that, although not widened, are systematically shifted to higher or lower apparent magnitudes relative to the central sub-field, depending on whether they trace the nearby or the faraway part of the tidal stream. With accurate 

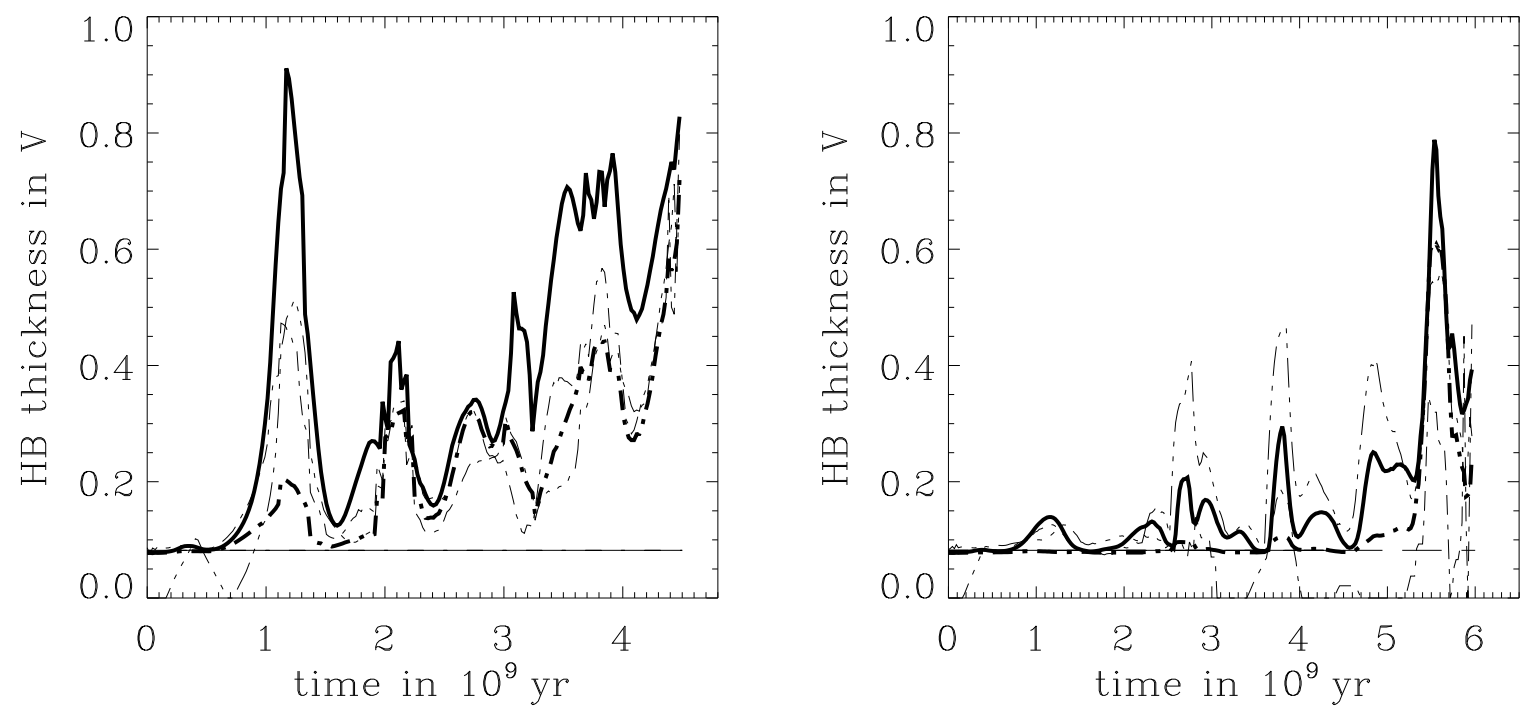

FIG. 4.- Time evolution of the horizontral branch (HB) thickness in V for models Sat-M1 and Sat-M2. The HB thickness is determined as the average over

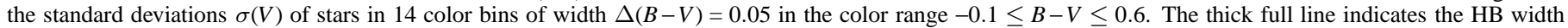

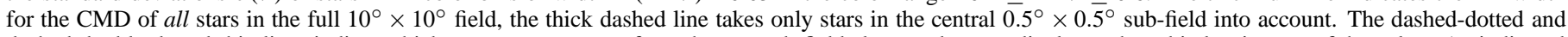

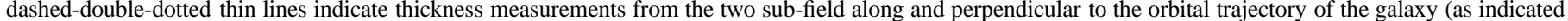

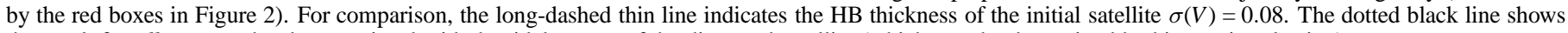
the result for all stars on the sky associated with the tidal stream of the disrupted satellite (which may be determined by kinematic selection).

photometry, this opens the possibility to still test the tidal scenario, even without a wide-field survey, by taking small fields along the major axis of the satellite and comparing the mean apparent magnitudes of the HB stars. Any systematic variation with position along the axis may indicate distance moduli variations due to the tidal effect. However, field contamination by Galactic foreground stars may pose a possible observational problem. Especially the red part of the HB appears in a region of the CMD that may be populated by halo turnoff stars. The blue part of the HB is less susceptible to this effect, but even there, some field stars are to be expected.

The time dependence of the HB thickness for both satellites is shown in Figure 4 . After the perigalactic passage that completely dissolves each satellite, i.e. after $t \gtrsim 1.5 \times 10^{9} \mathrm{yr}$ for Sat$M 1$ and $t \gtrsim 5 \times 10^{9} \mathrm{yr}$ in case of Sat-M2 (see KK98 for details), the $\mathrm{HB}$ of the satellite remnant becomes permanently and significantly widened compared to the bound stage. The HB width in the full field tends to be about 1.5 to 2 times larger than in the individual sub-fields. If dwarf galaxies are required to move on orbits with eccentricities larger than considered here in order to reach better morphological agreement with the observations, then the estimates for the expected HB width derived in this paper have to be seen as lower limits.

\section{RADIAL VELOCITY}

The relation between distance modulus and position along the semi-major axis of the dSph galaxy (Figure 21) introduces a similar correlation between the line-of-sight (LOS) velocity and position, as illustrated in Figure 5. Projecting the tidal debris of the disrupted satellite into the observer's sky leads to a significant LOS gradient along the orbital trajectory, simply because for larger distances from the stellar density maximum (along the tidal stream and subsequently on the plane of the sky) stars lag behind or advance ahead faster. This can be determined in a spectroscopic survey along the major axis of the dSph galaxy, as for typical projections the orbital path corresponds to the major axis of the galaxy. Only for extremely small viewing angles with respect to the tangent to the trajectory it is possible for a terrestrial observer to obtain projections of the orbital path onto the minor axis. This, however, requires very high orbital eccentricities and appears unlikely. The spectroscopic probe of the tidal scenario again requires covering large areas in order to be able to determine the LOS velocity gradient sufficiently well. The expected LOS velocity gradients are typically of the order of $20 \mathrm{~km} \mathrm{~s}^{-1}$ per degree (see also $\$ 4.2$ in K97).

\section{DISCUSSION AND SUMMARY}

Dwarf galaxies are excellent systems for testing current theories of, and alternatives to, dark matter. If they are dark matter dominated, their large mass-to-light ratios make the velocity dispersion profile or the rotation curve a clean measure of the dynamical mass in dark matter at all radii. Recent observations of gas-rich dwarfs show a nearly solid-body rotation curve, which implies a finite core inside a few hundred pc. This is inconsistent with cold dark matter (CDM) models (van den Bosch et al. 2000). Numerical simulations of CDM models invariably produces a diverging, power-law “cusp" of dark matter density $\rho \propto r^{-\gamma}$ with $\gamma=1.25 \pm 0.25$ at the scale of a few hundred pc (Navarro, Frenk \& White 1996, Moore et al., 1998). This failure of CDM also cannot be rescued by violent feedback from star formation and supernovae (Gnedin \& Zhao 2001). The CDM models are furthermore problematic because they predict the formation of too many dwarf satellites for a MilkyWay-sized galaxy. These problems are some of the driving motivations to look for alternatives to CDM. Many people have taken this as a need to assign new properties to the dark matter (self-interacting dark matter, warm dark matter etc.), or to modify the Newtonian law of gravity. Here we reexamined the 

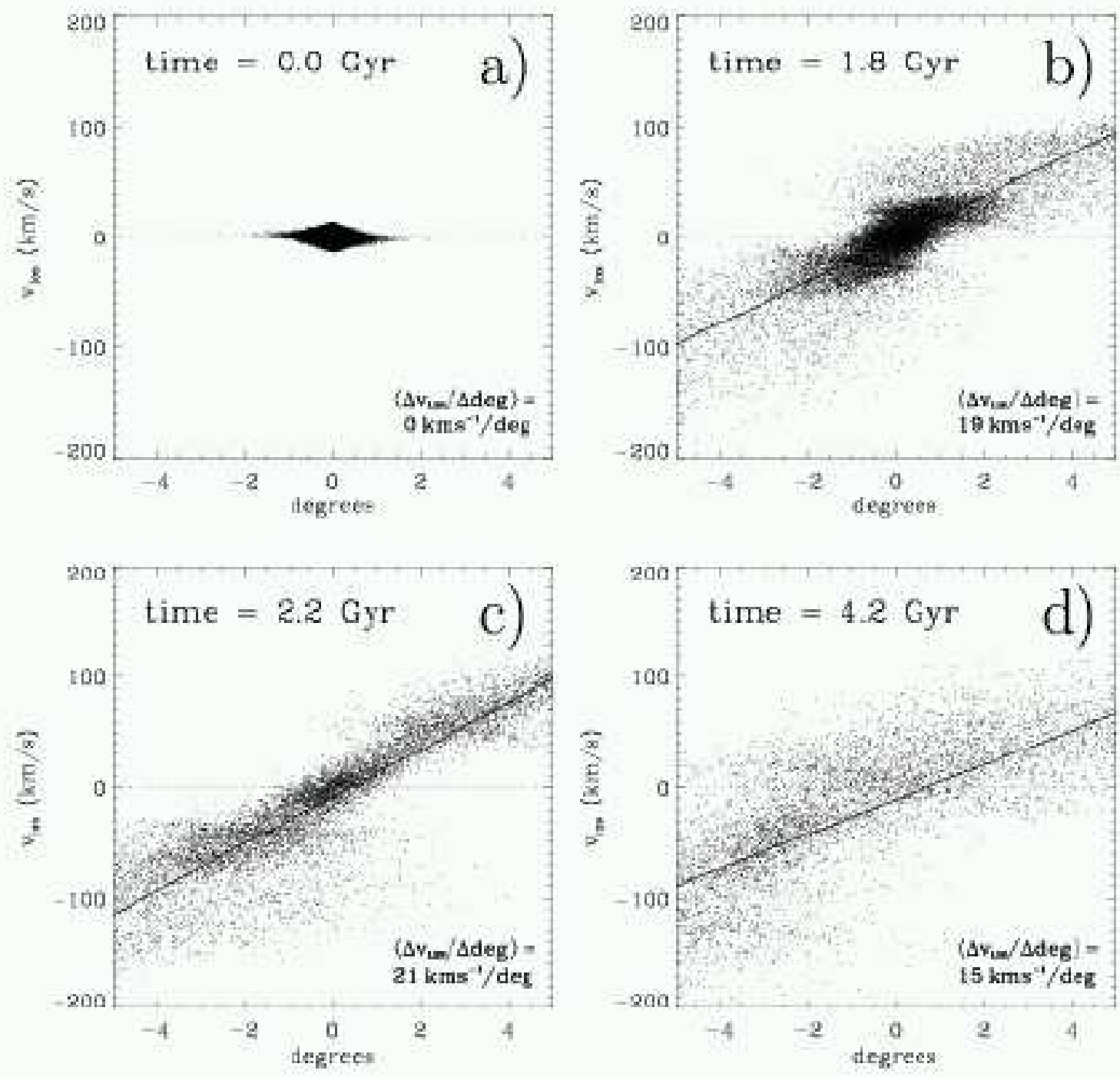

FIG. 5.- Line-of-sight (LOS) velocity gradient for the model dwarf galaxy Sat-M1 at four different times of its evolution. The LOS velocity is plotted versus position for stars selected in a slice of width $0.5^{\circ}$ along the major axis of the galaxy. The selected times are identical to Figure 2 The tidal model predicts velocity gradients along the orbital trajectory, for typical projections this corresponds to the major axis of the galaxy. The expected LOS velocity gradients are of the order of $20 \mathrm{~km} \mathrm{~s}^{-1} /$ deg.

non-equilibrium models of dwarf spheroidal galaxies proposed earlier (K97, KK98). These models are the most conservative models which introduces neither non-Newtonian dynamics nor any dark matter. In the non-equilibrium models, the observed $\mathrm{dSph}$ galaxies are identified as the unbound remnants of tidally disrupted satellite galaxies on an eccentric orbit with two tidal arms extending along a small angle from the line of sight of a terrestrial observer. We demonstrated that these models predict a detectable spread in distance modulus and a radial velocity gradient during most of the evolution after tidal disintegration.

The depth effect could be detected with wide-field photometric imaging of the Galactic dSph galaxies. To achieve statistical significance it is necessary to survey a dwarf galaxy out to several half-mass radii. This is feasible with the recent widefield cameras (e.g. at the $2.2 \mathrm{~m}$ telescope of ESO at La Silla). Distance modulus variations are best studied with horizontal branch stars outside the instability strip as they are bright (and easily detectable for the Galactic dSph's) and have very similar luminosity over a wide range of colors and metallicities. If the Galactic dSph's are of tidal origin, then the width of the horizontal branch due to the distance effect is expected to exceed its intrinsic width significantly. The models furthermore predict a systematic variation of distance moduli with position across the galaxy. This can be used to discriminate the effect from HB widening due to stellar evolution and the star formation history, because these should not correlate with position.

The non-equilibrium models furthermore predict a strong line-of-sight velocity gradient along the orbital trajectory, hence radial velocity data of the Galactic $\mathrm{dSph}$ is needed not only to test the tidal scenario, but also to establish alternative dark matter models and ultimately the dark matter profiles in dwarfs.

Kleyna et al. (1998) for example tried to measure the distance gradient effect between two central fields within 30 arcmins of Ursa Minor. They found the projection angle is between $90^{\circ}$ (no gradient) to $17^{\circ} .5$. Unfortuately this is only a $1 \sigma$ result, and it would be interesting to extend the study to outer 
fields where the effect would be more prominent. New data coming from Draco (Aparicio, Carrera, \& Martínez-Delgado 2001) and Ursa Minor (Martínez-Delgado et al. 2001 - which confirms the double-centerness of Ursa Minor) begin to set the first constraints on the present model. No obvious variation of distance modulus is seen in high quality multiband INT WFC images of the central $40^{\prime} \times 40^{\prime}$ of Ursa Minor and Draco (M. Irwin 2001, private communication). There is an indication of velocity gradient over a similar sized field in the recently completed spectroscopic data set of Draco stars, with a slope of about $12 \mathrm{~km} \mathrm{~s}^{-1} / \mathrm{deg}$, but curiously orthogonal to the expected orbital path of Draco (J. Kleyna 2001, private communication, see however Figure $4 \mathrm{a}$ in K97). To further constrain the tidal models it may be ultimately necessary to carry out the difficult task of finding stars outside the main body of the dwarfs, and following up with spectroscopy. A certain number of extra-tidal stars is expected for these dwarfs, given the strong tidal forces they experience in the Milky Way halo.

We have reported results from two tidal models with quite typical physical parameters. It was the aim of this paper to illustrate the expected effects and suggest possible observational tests rather than to describe one of the observed dSph's in particular. The overall parameter space for the tidal models (e.g. satellite mass and density profile, orbital eccentricity and apogalactic distance) is very large. Although our general conclusions will remain valid, quantitative estimates of the HB thickness as well as direction and strength of a possible velocity gradient depend on the adopted initial conditions. Therefore applying the proposed test to Galactic dwarfs requires some readjustments, for example shifting of the off-center control fields (typically they can be placed closer to the center of surface den- sity as the observed dwarfs are somewhat less extended than the projections in our models). A more comprehensive quantitative analysis of the tidal model requires the statistical analysis of a large number of model calculations. This remains work for the future.

How will our result change if some amount of dark matter is present in the dwarf spheroids? Without further simulations, we suspect that our results remain qualitatively the same. Our present models may be generalized to included cold dark matter if we assume it follows the same profile as the stars. Indead, a small dark halo may not be enough to bind the system together. This is supported observationally by the nearly complete disruptions in the Sagittarius galaxy at $16 \mathrm{kpc}$ and the $\mathrm{SMC}$ at $60 \mathrm{kpc}$. The CDM models predict a virial radius of roughly $10 \mathrm{kpc}\left(\sigma / 10 \mathrm{kms}^{-1}\right)$ for a pristine NFW halo in the field, which is much bigger than the observed tidal truncation radius of the dwarfs in the Milky Way halo. So it is likely that the tidal shocks can significantly modify the density structure of a dwarf galaxy, both the stellar density profile and the profile of any dark matter halo.

We thank Josef Fried, Eva Grebel, Mike Irwin, Jan Kleyna, Pavel Kroupa, Doug Lin, Bryan Miller, \& Mark Wilkinson for stimulating discussions. And we grateful to the comments and suggestions by our referee Tad Pryor whose careful reading helped to improve and clarify the arguments presented here. RSK acknowledges support by a Otto-Hahn-Stipendium of the Max-Planck-Gesellschaft and subsidies from a NASA astrophysics theory program supporting the joint Center for Star Formation Studies at NASA-Ames Research Center, UC Berkeley, and UC Santa Cruz.

\section{REFERENCES}

Aparicio, A., Carrera, R., \& Martínez-Delgado, D. 2001, AJ, submitted

Bekki, K., Couch, W. J., Drinkwater, M. J. 2001, ApJ, 552, L105

Bounanno, R., Corsi, C. E., Buzzoni, A., Cacciari, C., Ferraro, F. R., Fusi Pecci, F. 1994, A\&A, 290, 69

de Blok, W. J. W., McGaugh, S. S., Bosma, A., \& Rubin, V. C. 2001, ApJ, 552, L23

Ebisuzaki, T. Makino, J., Fukushige, T., Taiji, M., Sugimoto, D., Ito, T., Okumura, S. 1993, PASJ, 45, 269

Gnedin, O., Zhao, H., 2001, MNRAS, submitted astro-ph/0108108)?

Grebel E. K. 1997, Reviews in Modern Astronomy, 10, 29

Helmi, A., White, S. D. M. 1999, MNRAS, 307, 495

Ibata, R. a., Girmore, G., \& Irwin, M. J. 1994, Nature, 370, 194

Irwin, M., Hatzidimitriou, D. 1995, MNRAS, 277, 1354

Johnston, K. V., Sigurdsson, S., Hernquist, L. 1999, MNRAS, 302, 771

Kleyna, J. Geller, M. Kenyon S., Kurtz, M., Thorstensen J. 1998, AJ, 115, 2359

Klessen, R. S., Kroupa, P. 1998, ApJ, 498, 143

Kroupa, P. 1997, New Aston., 2, 139
Kuhn, J. R. 1993, ApJ, 409, L13

Mateo, M. 1998, ARA\&A, 36, 435

Martínez-Delgado, D., Alonso-García____aparicio, A., \& Gómez-Flechoso, M. A. 2001a, ApJ, in press astro-ph/0101456

Martínez-Delgado, D., Gómez-Flechoso, M. A., Aparicio, A., \& AlonsoGarcía, J. 2001b, AJ, in preparation

Moore B. et al. 1998, ApJ, 499, L5

Navarro J.F., Frenk C.S., \& White S.D.M., 1996, ApJ, 462, 563

Oh, K. S., Lin, D. N. C., Aarseth, S. J. 1995, ApJ, 442, 142

Piatek, S., Pryor, C. 1995, AJ, 109, 1071

Sandage, A. R. 1953, AJ, 58, 61

Sandage, A. R., Katem, B. 1982, AJ, 88, 1146

Sugimoto, D., Chikada, Y., Makino, J., Ito, T., Ebisuzaki, T., Umemura, M. 1990, Nature, 345, 33

van den Bosch, F. C., Robertson, B. E., Dalcanton, J. J., \& de Blok, W. J. G. 2000, AJ, 119, 1579 